Reviews in Mathematical Physics

Vol. 24, No. 10 (2012) 1292001 (2 pages)

(C) World Scientific Publishing Company

DOI: $10.1142 /$ S0129055X12920018

ERRATA

\title{
A HOLOMORPHIC REPRESENTATION OF THE JACOBI ALGEBRA
}

[Reviews in Mathematical Physics, Vol. 18, No. 2 (2006) 163-199]

\section{STEFAN BERCEANU}

National Institute for Physics and Nuclear Engineering,

Department of Theoretical Physics,

PO Box MG-6, Bucharest-Magurele, Romania

Berceanu@theory.nipne.ro

Received 13 October 2012

Published 1 November 2012

On p. 177, Eq. (6.11) should read

$$
\tilde{A}=\left(\begin{array}{c}
A \\
\bar{A}
\end{array}\right) ; \quad \mathcal{D}=\mathcal{D}(z)=\left(\begin{array}{cc}
M & N \\
P & Q
\end{array}\right) .
$$

On p. 182, Eq. (6.44) should read

$$
\lambda=(\bar{a}+\bar{b} w)^{-2 k} \exp \left(\frac{z}{2} \bar{\alpha}_{0}-\frac{z_{1}}{2} \bar{\alpha}_{2}\right) \exp i \theta_{h}\left(\alpha_{1}, \alpha_{0}\right) .
$$

On p. 184, the equation of $(\phi, \psi)_{\mathcal{H}^{*}}$ after Eq. (7.3) should read

$$
(\phi, \psi)_{\mathcal{H}^{*}}=\left(f_{\phi}, f_{\psi}\right)_{\mathcal{F}_{\mathcal{H}}}=\frac{1}{\pi} \int \bar{f}_{\phi}(z) f_{\psi}(z) e^{-|z|^{2}} d \Re z d \Im z .
$$

On p. 185, the second equation for $e_{z, w}$ should read

$$
e_{z, w}=\sum_{n} \frac{i^{-n}}{(n !)^{1 / 2}} \mid n>\left(\frac{w}{2}\right)^{\frac{n}{2}} H_{n}\left(\frac{i z}{\sqrt{2 w}}\right) \sum_{m} \frac{w^{m}}{m ! a_{k^{\prime} m}} e_{k^{\prime}, k^{\prime}+m} .
$$

On p. 185 , the polynomial $P_{5}$ in Eq. (7.12) should be

$$
P_{5}=z^{5}+10 z^{3} w+15 z w^{2} .
$$

On p. 187, the expression of $f_{w \bar{w}}$ has the value

$$
f_{w \bar{w}}=\frac{A^{\prime \prime}}{2(1-w \bar{w})^{4}}+\frac{2 k}{(1-w \bar{w})^{2}} .
$$


Errata

On p. 189, Eqs. (8.6) should read

$$
\begin{aligned}
i \dot{z} & =\epsilon_{a}+\frac{\epsilon_{0}}{2} z+\bar{\epsilon}_{a} w+\epsilon_{+} z w, \\
i \dot{w} & =\epsilon_{-}+\epsilon_{0} w+\epsilon_{+} w^{2} .
\end{aligned}
$$

